Relations industrielles

Industrial Relations

\title{
Morin, Marie-Laure, Le droit des salariés à la négociation collective : principe général du droit
}

\section{Pierre Verge}

Volume 50, numéro 4, 1995

L'ergonomie et les relations industrielles

Ergonomics and Industrial Relations

URI : https://id.erudit.org/iderudit/051061ar

DOI : https://doi.org/10.7202/051061ar

Aller au sommaire du numéro

\section{Éditeur(s)}

Département des relations industrielles de l'Université Laval

\section{ISSN}

0034-379X (imprimé)

1703-8138 (numérique)

Découvrir la revue

Citer ce compte rendu

Verge, P. (1995). Compte rendu de [Morin, Marie-Laure, Le droit des salariés à la négociation collective : principe général du droit]. Relations industrielles /

Industrial Relations, 50(4), 890-893. https://doi.org/10.7202/051061ar

Tous droits réservés @ Département des relations industrielles de l'Université Laval, 1995
Ce document est protégé par la loi sur le droit d'auteur. L'utilisation des services d'Érudit (y compris la reproduction) est assujettie à sa politique d'utilisation que vous pouvez consulter en ligne.

https://apropos.erudit.org/fr/usagers/politique-dutilisation/ 
se dégage en filigrane dans tout leur ouvrage constitue toutefois une avenue à privilégier pour remédier à certaines des limites actuelles du régime: les auteurs s'élèvent contre une trop grande judiciarisation du régime et contre l'importation de concepts et de techniques inappropriés de droit civil ou de common law, l'arbitrage de grief devrait selon eux se détacher des approches traditionnelles et civilistes de la convention collective, pour la comprendre et la saisir d'une manière plus concrète, plus souple et plus adaptée aux besoins des parties, en privilégiant une approche systémique, à l'opposé des exégèses.

En conclusion, on ne peut donc que souligner le caractère indispensable de cet ouvrage pour tous les praticiens de l'arbitrage de grief et sa contribution majeure à la doctrine.

ClaudeTte RosS

Université du Québec à Montréal

\section{Le droit des salariés à la négociation collective : principe général du droit} par Marie-Laure MORIN, Paris, L.G.D.J., 1994, 681 p., ISBN 2-275-00420-3 et ISSN 0520-0180.

Version actualisée d'une thèse de doctorat, l'imposant ouvrage de madame Morin, chargée de recherche, CNRS, au Centre d'études juridiques et économiques de l'emploi de l'Université de sciences sociales de Toulouse, présente le droit français de la négociation collective, non pas d'une façon linéaire, mais à travers la démonstration de sa nature essentielle. Son titre révèle simplement le principe qui irradie ce droit: un véritable droit des salariés à la négociation collective, principe général du droit. Ce droit des salariés "à la négociation collective de l'ensemble de leurs conditions de travail et d'emploi et de leurs garanties sociales ", qu'affirme la Loi du 13 juillet 1971 , se retrouve aujourd'hui en l'article L. 131-1 du Code du travail, à la tête de l'ensemble de ses règles d'exercice ; il traduit l'affirmation constitutionnelle de 1946 (alinéa 8 du Préambule de la Constitution): "Tout travailleur participe par l'intermédiaire de ses délégués à la négociation collective des conditions de travail ainsi qu'à la gestion des entreprises ".

Il s'agit bien là d'un principe général du droit, notion dont l'auteure établit préalablement et théoriquement les exigences et la portée. Par sa place hiérarchique, par sa généralité d'extension, un tel principe "assure la cohérence des institutions positives qui le matérialisent et l'évolution du système juridique dans son environnement (naissance de nouvelles normes, transformation interne du système juridique, etc.)" (p. 58).

Conforté par l'évolution du droit international (cf. les conventions $\mathrm{n}^{\circ} 87$ et 98 de l'OIT notamment et le développement plus récent du droit européen), l'actuel droit des salariés à la négociation collective est édifié en droit interne, comme le démontre la première partie de l'ouvrage, sur l'affirmation progressive de la négociation collective : conception d'abord contractuelle de la négociation collective (loi du 25 mars 1919), régime de la convention étendue de la loi de 1936, qui fait de la branche, de la profession, le niveau central de négociation, reconnaissance de la capacité normative des parties à différents niveaux de négociation résultant de la loi de 1950, généralisation de l'autonomie collective, non pas au sens absolu du droit allemand, mais sous l'égide de la loi. Ce droit de négocier qu'affirme la loi de 1971 est donc devenu un droit général de négocier : accords d'entreprises, conventions nationales locales ou régionales, de portée sectorielle, ou même accords interprofessionnels. Centré jusque-là sur le négocié, droit de l'accord, le droit à la négociation allait par la 
suite prendre également une dimension procédurale, devenir aussi un droit de la négociation: le législateur de 1982, en particulier, reconnait spécifiquement le droit à la négociation collective dans l'entreprise (C.t., art. L. 132-18) et l'accompagne d'une obligation périodique de négocier.

Le droit à la négociation en contexte français s'entend donc d'un droit pouvant s'exercer de façon complémentaire à différents niveaux, selon la diversité des intérêts collectifs des salariés en jeux: "La négociation collective n'est plus d'abord le moyen d'organiser la profession, elle est un moyen pour les salariés, à tous les niveaux, de participer à la détermination de leurs conditions de travail, à partir de la relation de travail subordonnée qui est la leur dans l'entreprise " (p. 245).

Ainsi façonné et affirmé, il s'agit maintenant de voir comment ce droit se réalise en droit positif, objet de la seconde partie de l'ouvrage : il est bien un droit des salariés, même si des syndicats en sont exclusivement les agents. L'appartenance des salariés à une même collectivité leur confère un intérêt collectif à la mesure de celle-ci : entreprise, branche... C'est cette situation collective qui justifie une égalité de traitement des salariés au sein de la collectivité; de là, l'effet impératif de la convention et, surtout, sa portée erga omnes à l'intérieur du groupe visé. Tout salarié a droit au fruit de l'accord en raison de sa seule appartenance à la collectivité. Cet effet s'impose indépendamment de tout choix syndical individuel : "il s'agit donc bien d'un droit collectif "(p. 281). L'appartenance simultanée des salariés à une pluralité de collectivités de négociation supposera une coordination des accords de différents niveaux. Droit univoque, à la différence des droits nordaméricains, seuls les salariés en sont titulaires, à l'exclusion de l'employeur. Droit des salariés à la négociation, mais non pas droit de négocier; ce pouvoir est dévolu, dans la tradition française aux syndicats considérés comme représentatifs par l'État: " dimension proprement politique et cette notion, conçue à l'origine pour permettre l'association des partenaires sociaux à l'action de l'État " (p. 361). Effet erga omnes de la convention et exigence de représentativité syndicale, voilà deux éléments caractéristiques du droit des salariés à la négociation: la fonction réglementaire de l'accord " justifie l'exigence de représentativité des syndicats signataires, telle que [le droit français] la conçoit et la nature de leurs pouvoirs " (p. 608).

Application du principe de participation, le droit des salariés à la négociation collective se traduit de plus en plus en des règles de procédure régissant, à divers niveaux, la prise de décisions touchant leurs conditions de travail. Tantôt la loi impose directement à l'employeur (ou à un groupement d'employeurs) de négocier relativement à différents sujets. Ainsi, parmi différentes prescriptions, le Code (art. L. 132-12) oblige-t-il les partenaires sociaux à examiner tous les cinq ans la nécessité de réviser les classifications; existe également une obligation de négocier la formation professionnelle, qui concerne d'abord les branches professionnelles (art. L. 933-2, p. 458). Une négociation périodique de branche portant d'abord sur les salaires minimums a cours (art. L. $132-12$, p. 462). Dans l'entreprise, une négociation annuelle s'impose relativement aux salaires effectifs, à la durée et à l'organisation du temps de travail et à la situation de l'emploi dans l'entreprise (art. L. 132-27, p. 464). Tantōt, la négociation se présentera comme une condition d'exercice du pouvoir de direction de l'entreprise. Différentes procédures de négociation ont ainsi trait à des dérogations aux dispositions légales ou réglementaires en matière d'aménagement du temps de travail. Le droit à la négociation trouvera aussi des manifestations jurisprudentielles, notamment s'agissant de la dénonciation d'un usage d'entreprise. L'auteure examine aussi la 
place limitée de la négociation dans les conflits collectifs, les " rapports complexes " (p. 49l) qu'entretiennent le droit à la négociation et la liberté constitutionnelle de grève des salariés. La procédure d'extension des conventions collectives peut également être vue comme une manifestation du droit à la négociation (p. 510). Le droit à la négociation se manifeste, certes, dans toutes ces situations particulières, mais le principe en cause doit surtout conduire au constat suivant : "S'il n'est pas mutuellement défini comme un droit général d'obtenir des négociations, en dehors des obligations déterminées de négocier, il nourrit et explique certaines solutions positives, et fournit une base pour obtenir des négociations (usage), apprécier le comportement de l'employeur (conflit du travail), ou justifier l'intervention de la puissance publique " (p. 553). Sa réalisation "doit se mesurer à l'aune des institutions positives qu'il coordonne, mais c'est un droit "ouvert" qui peut se manifester autrement et dont la réalisation se poursuit " (ibidem).

L'exercice du droit à la négociation porte le législateur à formuler des règles objectives de déroulement, parfois fort précises, comme lorsqu'il s'agit de la négociation d'entreprise - par exemple, l'obligation pour les négociateurs de fixer le calendrier des réunions (art. L. 132$22)$; il laisse ainsi relativement peu de place à l'appréciation globale des comportements des parties qui négocient, comme dans les droits nord-américains. L'auteure se penche également sur les sanctions du droit à la négociation.

Un dernier chapitre, fort substantiel, examine enfin les fonctions du droit à la négociation. La reconnaissance du droit de négocier collectivement "[...] n'a jamais signifié abstention de l'Êtat dans le domaine de la négociation collective. Il y a là une caractéristique du système français " (p. 556) : omniprésence de la loi d'ordre public, "intimement liée à la faiblesse traditionnelle de la négociation [en France] " (ibidem). La convention collective a pour fonction traditionnelle d'améliorer la situation des salariés; elle ne peut déroger (art. L. 132-4) aux avantages qu'ils tiennent de la loi. II en va de même du contrat individuel par rapport à la convention collective, sujet que l'auteure étudie aussi méthodiquement. La notion d'ordre public social joue un rôle central. Mais (cf. les dérogations négociées ou même, la loi négociée elle-même), l'évolution va dans le sens d'une " interpénétration de plus en plus grande des normes légales et des normes conventionnelles dans la régulation des relations de travail $[\ldots]$ " (p. 607): langage de la flexibilité, également applicable à la position hiérarchique des accords collectifs de différents niveaux entre eux. L'essor de la négociation d'entreprise tait aussi voir que le "droit à la négociation n'est plus seulement le droit de négocier le statut de base des salariés, il est aussi un instrument d'adaptation des entreprises dans la mutation économique et technologique " (p. 592). En somme, "la physionomie de l'ordre public social se transforme " (p. 631). Le tout ne va pas sans interrogations. Ainsi, la négociation d'entreprise doit-elle continuer d'être soumise au régime général de la négociation collective ou ne doitelle pas être traitée de façon particulière (p. 637) ? La notion de représentativité syndicale est aussi interpellée: "peutelle perdurer alors qu'elle ne repose, dans l'entreprise, que sur un minimum de présence syndicale " (ibidem)?

L'édifice français de la négociation collective est une construction complexe. Le contenu de l'ouvrage est à sa mesure et la précédente évocation de son contenu ne saurait lui rendre justice. Bien que centré sur le droit français et extrêmement bien documenté, tant du point de vue doctrinal que jurisprudentiel (on ne pourrait que souhaiter plus de précision dans les renvois infrapaginaux à du matériel précité), il contient aussi des aperçus de certains aspects d'autres droits nationaux de la négociation collective. 
Si les droits nord-américains de la négociation collective, dont les droits canadiens et québécois, entretiennent depuis fort longtemps une conception intense de la négociation d'entreprise et du droit à celle-ci, le droit français impose, par ce droit général des salariés à la négociation collective, la "démocratie économique et sociale ", à côté de la démocratie politique dans le pays. Cette conception englobante de l'ordre social parait étrangère au droit canadien.

PIERRE VERGE

Université Laval

\section{Industrial Relations Under Liberal Democracy}

by Roy J. ADAMS, Columbia, S.C., University of South Carolina Press, 1995, 219 p., ISBN 1-57003-019-7.

This book has two purposes. The first is to delineate and explain the essential differences between the industrial relations (IR) systems of North America and those of other developed liberal democracies. The second is to draw lessons for the U.S. and Canada, based upon these differences and the performance outcomes associated with them.

The book begins with a short chapter on the early history of IR institutions, and of the "strategic choices" faced by labour movements at the end of the nineteenth century. This chapter is very sketchy and for the most part covers familiar ground, but it provides a nice set-up for the remainder of the book.

Chapters two and three address, respectively, the "European mainstream" and "North American exceptionalism," essentially documenting the different trajectories taken by the European and the North American IR systems from the late 1800 s to the present. In discussing the European mainstream, the author focuses upon Germany and Sweden, maintaining that the development of powerful socialist movements in these countries during the early 1900 s forced employers and states to forge historical compromises which recognized the right of centralized labour confederations to represent and bargain on behalf of all workers, while largely excluding unions from direct involvement in the management of work organizations. In contrast, the "socialist threat" was never as strong in North America, so neither employers nor the state found it necessary to forge a similar compromise. Instead, a more narrowly defined and decentralized system developed, in which workers formally enjoy the right to organize and bargain with their employers, but in which the legitimacy and influence of labour unions has been tenuous. In discussing these developments, Adams draws extensively upon the work of Selig Perlman and Adolf Sturmthal, but concludes that neither is entirely satisfactory in accounting for American exceptionalism.

Chapter four is perhaps the most valuable in the book, comparing the Canadian and U.S. systems with their European counterparts, while acknowledging differences between individual nations. This chapter provides an interesting and in some places a provocative analysis, identifying similarities and differences in the coverage, structure, scope, and processes of collective bargaining, as well as in strike laws and dispute resolution procedures.

Adams continues his comparative analysis in the following two chapters, with chapter five focusing upon differences in union densities and bargaining coverage, and chapter six focusing upon differences in socioeconomic performance. While acknowledging some positive attributes of the North American variant, he considers the North American system to be inferior in a number of areas, including bargaining coverage, 